\begin{tabular}{|c|c|}
\hline Title & Sclerostin Enhances A dipocyte Differentiation in 3T З-L1 Cells \\
\hline Author(s) & U kita, May umi; Y amaguchi, Taihiko; Ohata, Noboru; T amura, Masato \\
\hline Citation & $\begin{array}{l}\text { Journal of cellular biochemistry, 117(6), 1419-1428 } \\
\text { https://doi.org/10.1002/cb.25432 }\end{array}$ \\
\hline Issue Date & 2016-06 \\
\hline Doc URL & http:/hdl.handle.net/2115/65842 \\
\hline Rights & $\begin{array}{l}\text { This is the peer reviewed version of the following article: [Sclerostin enhances adipocyte differentiation in } 3 T \text { 3L1 } \\
\text { cells], which has been published in final form at [http://dx. doi.org/10.1002 } / \text { cb.25432]. This article may be used for non- } \\
\text { commercial purposes in accordance with Wiley Terms and Conditions for Self-A rchiving. }\end{array}$ \\
\hline Type & article (author version) \\
\hline File Information & Ukita_HUSCAP.pdf \\
\hline
\end{tabular}

Instructions for use 


\section{Sclerostin enhances adipocyte differentiation in 3T3-L1 cells}

Mayumi Ukita ${ }^{1,2}$, Taihiko Yamaguchi ${ }^{2}$, Noboru Ohata ${ }^{2}$, Masato Tamura ${ }^{1^{*}}$

${ }^{1}$ Department of Biochemistry and Molecular Biology, Graduate School of Dental Medicine, Hokkaido University, Sapporo, 060-8586, Japan

${ }^{2}$ Department of Crown and Bridge Prosthodontics, Graduate School of Dental Medicine, Hokkaido University, Sapporo, 060-8586, Japan

Running title: Sclerostin enhances adipocyte differentiation

Key word: sclerostin, adipocyte, osteocyte, Sost

Total page of text: 26 , figures 5 , table 1

Contract grant sponsor: None; Contract grant number: None.

"Corresponding Author: Masato Tamura, PhD.

Professor and Chairman

Department of Biochemistry and Molecular Biology

Graduate School of Dental Medicine

Hokkaido University

North 13, West 7, Sapporo 060-8586, Japan

Phone and Fax: 011-81-11-706-4231

E-mail:mtamura@den.hokudai.ac.jp 


\section{ABSTRACT}

Sclerostin, a secreted protein encoded by the Sost gene, is produced by osteocytes and is inhibited by osteoblast differentiation and bone formation. Recently, a functional association between bone and fat tissue has been suggested, and a correlation between circulating sclerostin levels and lipid metabolism has been reported in humans. However, the effects of sclerostin on adipogenesis remain unexplored. In the present study, we examined the role of sclerostin in regulating adipocyte differentiation using 3T3-L1 preadipocytes. In these cells, sclerostin enhanced adipocyte-specific gene expression and the accumulation of lipid deposits. Sclerostin also upregulated CCAAT/enhancer binding protein $\beta$ expression but not cell proliferation and caspase-3/7 activities. Sclerostin also attenuated canonical Wnt3a-inhibited adipocyte differentiation. Recently, the transcriptional modulator TAZ has been involved in the canonical Wnt signaling pathways. Sclerostin reduced TAZ-responsive transcriptional activity and TAZ-responsive gene expression. Transfection of 3T3-L1 cells with TAZ siRNA increased the lipid deposits and adipogenic gene expression. These results show that sclerostin upregulates adipocyte differentiation in 3T3-L1 cells, suggesting a possible role for the osteocyte-derived sclerostin as a regulator of fat metabolism and as a reciprocal regulator of bone and adipose tissues metabolism. 


\section{Introduction}

The existence of reciprocal regulation between bone and energy metabolisms is demonstrated by recent several reports [Karsenty and Oury, 2012]. Adipocytes play critical roles in the maintenance of energy balance, and these cells store energy in the form of lipids and release fatty acids in response to metabolic signals or to energy insufficiency [Ali et al., 2013]. Adipocytes are also known as endocrine cells that secrete a number of adipocytokines [Kadowaki and Yamauchi, 2005]. Among them, two adipocyte-derived secreted molecules, adiponectin and leptin, which are specifically and highly expressed in the adipose tissue and abundantly secreted into the blood, are known to regulate bone mass, and bone could be a target tissue for these hormones [Kajimura et al., 2013; Takeda et al., 2002]. In contrast, bone-derived molecules, which regulate adipocytes, have not been investigated yet. It was only reported that the osteoblast-specific secreted molecule osteocalcin behaves as a hormone regulating glucose metabolism and fat mass in mutant mice [DiGirolamo et al., 2012; Ferron et al., 2008]. However, it remains to elucidate which molecules secreted by bone cells can affect fat metabolism.

Sclerostin (the Sost gene product) is a $29 \mathrm{kDa}$ secreted protein of 213 amino acids characterized as a negative regulator of bone formation [Baron and Kneissel, 2013; Ke et al., 2012; van Bezooijen et al., 2005]. In the adult bone, Sost is constitutively expressed by osteocytes, final differentiated cells of the osteoblast lineage [Burgers and Williams, 2013; van Bezooijen et al., 2005]. Their role was first appreciated when excessive bone mass was observed in patients with sclerosteosis or Van Buchem's disease, an autosomal recessive disease with mutations or deletions in the Sost gene [Balemans et al., 2001]. Furthermore, a high bone mass phenotype was observed in sclerostin-null mice [Li et al., 2008] and a low bone mass phenotype in sclerostin-overexpressing mice [Kramer et al., 2010]. Sclerostin was detectable in 
serum in all healthy human subjects studied, suggesting that the protein is secreted and enters the circulation. From several data on circulating sclerostin serum levels in humans, a positive correlation was reported between circulating sclerostin and the percentages of abdominal fat, gynoid fat, and fat mass [Amrein et al., 2012; Klangjareonchai et al., 2014; Urano et al., 2012]. Consequently, it was suggested that sclerostin regulates adipogenesis or fat production. To date, little is known on the regulation of adipocyte differentiation in response to sclerostin.

During adipocyte differentiation, committed preadipocytes undergo growth arrest and subsequent terminal differentiation into adipocytes. In 3T3-L1 preadipocytes, growth-arrested cells have been shown to re-enter the cell cycle synchronously and to undergo mitotic clonal expansion in response to differentiation inducer treatment (a combination of 3-isobutyl-1-methylxanthine; [IBMX], dexamethasone, and insulin), before exiting the cell cycle and terminally differentiating [MacDougald and Lane, 1995]. Many transcription factors act sequentially during the differentiation processes [Rosen and Spiegelman, 2000]. Among them, CCAAT/enhancer binding protein (C/EBP) $\beta$ is a key transcription factor transcribed, phosphorylated, and activated immediately after exposure to the differentiation inducer treatment, thus resulting in the transactivation of C/EBPa and peroxisome proliferator-activated receptor (PPAR) y [Guo et al., 2015]. C/EBPa and PPARY can initiate differentiation, and acquisition of the adipocyte phenotype is characterized by an increase in the expression of adipocyte-specific genes such as lipoprotein lipase (LPL) [Rosen and Spiegelman, 2000]. Several hormones and growth factors that affect adipocyte differentiation in a positive or negative manner have been identified. Growth hormone and insulin like growth factor 1 stimulates adipogenesis. In contrast, the epidermal growth factor, transforming growth factor (TGF)- $\alpha$, TGF- $\beta$, and retinoic acid are generally considered inhibitors of adipocyte differentiation [Rosen and Spiegelman, 2000]. Also, canonical Wnt ligands are known to 
inhibit differentiation [Ross et al., 2000].

The effects of Wnt ligands on the canonical signaling pathway involving $\beta$-catenin are mediated by their binding to the Frizzled receptor and to coreceptors, low-density lipoprotein receptor-related proteins (LRPs) 4/5/6 [MacDonald et al., 2009]. Sclerostin was reported as an antagonist of the canonical Wnt signaling pathway by binding to the extracellular domain of LRP4/5/6 and disrupting Wnt-induced Frizzled-LRP complex formation [Baron and Kneissel, 2013; Li et al., 2005]. Canonical Wnt signaling causes stabilization of $\beta$-catenin, which then translocates into the nucleus, where it interacts with transcription factors including the lymphoid enhancing factor 1 and T-cell factors (TCFs) that regulate the expression of several target genes [MacDonald et al., 2009]. TAZ is a transcriptional coactivator originally identified in a proteomic screening for 14-3-3 binding protein [Kanai et al., 2000] and is well known to be regulated by the Hippo signaling pathway [Piccolo et al., 2014]. Recently, Azzolin et al. [Azzolin et al., 2012] reported TAZ as a downstream component of the canonical Wnt signaling pathway and as a mediator of Wnt biological responses independent of the Hippo pathway. It has been proposed that the canonical Wnt pathway induces TAZ protein stabilization and transcriptional activity in multiple cell types [Azzolin et al., 2014; Azzolin et al., 2012].

In the present study, we investigated whether osteocyte sclerostin regulates adipocyte differentiation. We found that sclerostin enhances adipocyte differentiation in 3T3-L1 cells and reduced TAZ-responsive transcriptional activity and TAZ-responsive gene expression, indicating a role for TAZ as a regulator of adipogenesis by sclerostin. 


\section{Materials and Methods}

\section{Reagents}

IBMX and dexamethasone were purchased from Sigma-Aldrich (St. Louis, MO). Insulin was purchased from Cell Science and Technology Institute Inc. (Sendai, Japan). Recombinant mouse sclerostin and Wnt3a were purchased from R\&D Systems (Minneapolis, MN).

\section{Cell cultures}

3T3-L1 cells were obtained from the DS Pharma Biomedical Inc. (Osaka, Japan) and grown to confluence in Dulbecco's modified Eagle's medium (DMEM, Sigma-Aldrich) with $100 \mu \mathrm{g} / \mathrm{mL}$ of kanamycin (Meiji, Tokyo, Japan) and 10\% fetal bovine serum (FBS; SAFC Bioscience, Inc., Lenexa, KS) at $37^{\circ} \mathrm{C}$ in a humidified atmosphere of $5 \% \mathrm{CO} 2$. For adipocyte differentiation, at two days postconfluence (day 0), differentiation was induced using the differentiation inducer treatment $(500 \mu \mathrm{M}$ IBMX, $10 \mu \mathrm{g} / \mathrm{mL}$ insulin and $1 \mu \mathrm{M}$ dexamethasone) added to a basal medium. At day 3, the medium was replaced with adipogenic medium containing DMEM supplemented with $10 \%$ FBS and $10 \mu \mathrm{g} / \mathrm{mL}$ insulin, which was changed every two days thereafter until analysis.

\section{Oil red $O$ staining}

3T3-L1 cells were washed with phosphate-buffered saline (PBS) and fixed with 10\% formalin for one hour at room temperature. The cells were then rinsed with $60 \%$ isopropanol. Oil red O $(0.12 \%$, Sigma-Aldrich) was added and incubated for 10 min with gentle agitation, followed by further washing with PBS. The dishes were subsequently scanned to get the pictures. Quantification of oil red O staining was performed by eluting the stain from the cells using $100 \%$ isopropanol and then quantifying the absorbance $(520 \mathrm{~nm})$ of the staining against a blank (100\% isopropanol) on a spectrophotometer 
(Hitachi U-1500, Tokyo, Japan).

\section{Reverse transcription-polymerase chain reaction (RT-PCR)}

Total RNA was extracted from the cells using Isogen (Nippongene, Toyama, Japan) as described previously [Nakashima et al., 2005]. RT-PCR was performed as previously described [Nakashima et al., 2005]. The primer sequences for each gene are shown in the Table. To account for any difference in the amount of RNA, $\beta$-actin was chosen as the endogenous control. The amplification products were separated by electrophoresis on $2 \%$ agarose gels.

\section{Quantification of gene expression by quantitative RT-PCR (qRT-PCR)}

The qRT-PCR was performed using assay-on-demand TaqMan probes (Applied Biosystems, Foster City, CA) and the StepOne ${ }^{\circledR}$ real time PCR system according to the manufacturer's protocol as previously described [lizuka et al., 2014]. The relative level of gene expression was quantified using the comparative $C_{T}$ method with $\beta$-actin or glyceraldehyde-3-phosphate dehydrogenase (GAPDH) expression as the endogenous control.

\section{Transfection of small interfering RNA (siRNA)}

3T3-L1 cells were transfected with Silencer select predisigned siRNA for the TAZ (Ambion, ID number s97145) gene or with Silencer negative control siRNA \#1 (Ambion) at a concentration of $10 \mathrm{nM}$ using Lipofectamine RNAiMAX (Invitrogen, Carlsbad, CA) according to the manufacturer's instructions as described previously [Uyama et al., 2012].

Western blot analysis 
Cells were washed with ice-cold PBS and suspended in CelLytic-M Mammalian cell lysis/extraction reagent (Sigma) plus a protease inhibitor (Complete mini, Roche, Indianapolis, IN). Whole cell extracts were separated by $10 \%$ SDS polyacrylamide gel electrophoresis, and transferred to a PVDF membrane (Millipore, Bedford, MA). The membrane was probed with polyclonal antibodies raised to anti-C/EBPß (Bioss Inc., Woburn, MA), anti-TAZ (Bioss Inc.) or anti- $\beta$-actin antibodies (GeneTex, Irvine, CA) using the ECL prime detection system (GE lifesciences, Pittsburgh, PA) according to the manufacturer's instructions.

\section{Reporter constructs and assay for luciferase activity}

The 8xGTIIC-Lux luciferase reporter construct [Dupont et al., 2011], a synthetic luciferase sensor containing multimerized responsive elements of TEAD, the main DNA-binding cofactor of TAZ, was obtained from Addgene (34615; Cambridge, MA). The reporter assay was performed as described previously [Nakashima et al., 2005].

\section{Detection of DNA synthesis by chemiluminescent bromodeoxyuridine (BrdU) ELISA}

To measure cell proliferation, newly synthesized DNA of replicating cells was assayed by BrdU incorporation using a BrdU labeling and detection ELISA-kit (Cell Proliferation Biotrak ELISA System version 2, GE Healthcare) according to the manufacturer's instructions. Briefly, confluent 3T3-L1 cells were incubated in differentiation media and treated with sclerostin, and then cultured for further $24 \mathrm{~h}$. Then, BrdU was added to the cells. After $4 \mathrm{~h}$, cells were fixed and DNA denatured, then incubated with an antibody to BrdU conjugated with peroxidase $\left(60 \mathrm{~min}, 37^{\circ} \mathrm{C}\right)$. Immune complexes were detected by incubation with tetramethylbenzidine as substrate for $5 \mathrm{~min}$, the reaction was stopped with $\mathrm{H}_{2} \mathrm{SO} 4$ and absorption measured at $450 \mathrm{~nm}$ using a microplate reader (iMark, 
Bio-Rad).

\section{Measurement of caspase-3/7 activity}

Cellular enzymatic activities of caspase-3/7 were determined by a caspase colorimetric assay (Caspase-Glo 3/7 Assay Systems, Promega, Madison, MI) as described previously [lizuka et al., 2014]. Briefly, for each reaction, cells were lysed and incubated with a luminogenic substrate containing the DEVD sequence, which is cleaved by activated caspase-3/7. After incubation at room temperature for one hour, luminescence was quantified using a Mini Lumat LB 9506 luminometer (Berthold, Bad Wildbad, Germany).

\section{Generation of plasmid construct and over-expression of TAZ}

TAZ expression plasmid was generated as follows and designated as pTAZ. Mouse TAZ cDNA was amplified from 3T3-L1 cells cDNA using primers designed to flank the mouse TAZ open reading frame (forward 5'-GGTTCCAGCTCGTCAGTT-3', reverse 5'-GTGTGAGTACAAAGGCAG-3') using PrimeSTAR Max DNA polymerase (Clontech Laboratories, Inc. Mountain View, CA) according to the manufacturer's instructions. The PCR product was run on a $1 \%$ agarose gel, purified and subcloned into the pcDNA3 vector (Invitrogen) using the In-Fusion advantage PCR cloning kit (Clontech) by Kpnl and Xbal sites according to the manufacturer's instructions. Individual clones of transformed E.coli were isolated from agar plates and the nucleotide sequences of each plasmid were confirmed by DNA sequencing. 3T3-L1 cells were transfected with PTAZ or empty vector (pcDNA3) using ScreenFect A (Wako Pure Chemical Industries Ltd., Osaka, Japan) according to the manufacturer's instructions.

\section{Statistical analysis}


The data are reported as the mean \pm standard deviation of three independent experiments and were analyzed by Student's t-test;-values of $P<0.05$ were considered significant. 


\section{Results}

\section{Sclerostin positively regulates adipocyte differentiation in 3T3-L1 cells}

To evaluate a potential role for sclerostin on adipocyte differentiation, we used the 3T3-L1 cells, a well-characterized model system in vitro, which authentically reproduces adipogenesis including expression of adipogenic genes and morphological changes. Regulation of adipocyte differentiation was evaluated by the appearance of the adipocyte phenotype, especially the accumulation of visible lipid droplets determined by oil red $\mathrm{O}$ staining. Oil red O-stained cytoplasmic lipid droplets and absorbance of oil red $\mathrm{O}$ staining increased during adipogenic cultures in response to 5 $\mathrm{ng} / \mathrm{mL}$ of sclerostin and further augmented with increasing doses of sclerostin compared with untreated cells (Figs. 1A and 1B). The expression level of adiponectin and PPARY, which are known to be induced during adipogenesis [Rosen and Spiegelman, 2000], was increased in a dose-dependent manner by sclerostin, as determined by qRT-PCR (Fig. 1C). Not only adiponectin and PPARy, but also LPL and fatty acid-binding protein 4 (Fabp4) (also known as aP2) are known to be involved in adipocyte differentiation [Rosen and Spiegelman, 2000]. In sclerostin-stimulated 3T3-L1 cells, LPL and Fabp4 mRNA expression augmented with increasing doses of sclerostin during adipocyte differentiation, as determined by qRT-PCR (Fig. 1D). We also detected the enhancement of oil red $\mathrm{O}$ staining by sclerostin in presence of PPARY agonist rosiglitazone $(1 \mu \mathrm{g} / \mathrm{mL})$ in 3T3-L1 cells (data not shown). These findings indicate that sclerostin positively regulates adipocyte differentiation in 3T3-L1 cells.

\section{Sclerostin attenuates Wnt3a-inhibited adipocyte differentiation and expression of LRP5 and LRP6 in 3T3-L1 cells}

Canonical Wnt ligands are known to inhibit adipocyte differentiation. Treatment with Wnt3a, a canonical Wnt ligand, decreased adiponectin and PPARY mRNA gene 
expression in 3T3-L1 cells (Fig. 2A), as previously reported [Bennett et al., 2002; Ross et al., 2000]. Addition of sclerostin attenuated the Wnt3a-dependent reduction of adiponectin and PPARy mRNA expression (Fig. 2A). As expected, Wnt3a decreased the amount of oil red $\mathrm{O}$ staining to a comparable degree of the staining in cells differentiated in the presence of differentiation media alone. Wnt3a-mediated reduction of oil red $\mathrm{O}$ staining was increased by the addition of sclerostin (Fig. 2B), indicating that sclerostin attenuated the effect of canonical Wnt on 3T3-L1 cell differentiation.

Sclerostin has been reported to interfere with the canonical Wnt signaling pathway due to binding to the Wnt coreceptors LRP5/6 [Li et al., 2005] and LRP4 [Holdsworth et al., 2012]. Therefore, we examined which LRP may be expressed by 3T3-L1 cells. LRP5 and 6 mRNAs were detected in 3T3-L1 cells, whereas LRP4 mRNA expression could not be detected in these cells (Fig. 2C). Osteoblastic MC3T3-E1 cells expressed LRP4, 5, and 6 (Fig. 2C), as previously reported elsewhere [Choi et al., 2009].

\section{Sclerostin regulates C/EBP $\beta$ expression but not cell proliferation and caspase-3/7} activity in 3T3-L1 cells

The differentiation of 3T3-L1 cells into adipocytes is accompanied by a transient induction of $\mathrm{C} / \mathrm{EBP} \beta$, and overexpression of $\mathrm{C} / \mathrm{EBP} \beta$ has been shown to induce adipocyte differentiation [Guo et al., 2015]. Therefore, C/EBP $\beta$ expression was examined in response to sclerostin treatment. Sclerostin increased C/EBP $\beta$ protein level 24 hours after differentiation inducer treatment (Fig. 3A). However, DNA synthesis and caspase-3/7 activity were not altered by sclerostin treatment in 3T3-L1 cells (Figs. 3B and $3 \mathrm{C}$ ), indicating that the enhancement of adipocyte differentiation by sclerostin was not dependent on cell proliferation and apoptosis. 


\section{Regulation of TAZ activity by sclerostin}

Recently, canonical Wnt signaling has been reported to regulate direct transcriptional activation of responsive elements of TEAD, the main DNA-binding cofactor of the transcriptional coactivator TAZ [Azzolin et al., 2012]. Therefore, to explore the effect of sclerostin on this transcriptional activity, we transfected 3T3-L1 cells with 8xGTIIC-Lux [Dupont et al., 2011], a synthetic luciferase reporter containing multimerized responsive elements of TEAD. The induction of luciferase activity was observed after the treatment with Wnt3a, and sclerostin reduced the activity induced by Wnt3a (Fig. 4A). Sclerostin reduced the luciferase activity (Fig. 4A). Next, we examined the expression of the TAZ target gene ctgf [Zhang et al., 2009] in 3T3-L1 cells. Ctgf mRNA expression was induced by Wnt3a, whereas sclerostin inhibited Wnt3a-induced or not-induced ctgf expression (Fig. 4B). These results indicated that sclerostin downregulated TAZ activity in 3T3-L1 cells.

\section{Effects of TAZ knockdown or over-expression on sclerostin-mediated adipocyte differentiation in 3T3-L1 cells}

To evaluate the potential biological relevance of regulation of the TAZ pathway in sclerostin-mediated adipocyte differentiation, we examined the effect of TAZ knockdown using RNA interference. Following transfection of 3T3-L1 cells with TAZ siRNA, the protein level of TAZ diminished, confirming that the siRNA was effective in silencing endogenous TAZ expression (Fig. 4C). The increase of lipid accumulation was observed in TAZ siRNA-treated cells compared with control siRNA-treated 3T3-L1 cells (Fig. 4D), indicating that knockdown of TAZ induces adipocyte differentiation. Concomitant treatment with sclerostin and with TAZ siRNA enhanced oil red O staining and adiponectin mRNA expression compared with TAZ siRNA-treated cells (Figs. 4E and 4F). Next, we examined the effect of TAZ over-expression on sclerostin-mediated 
adipocyte differentiation in 3T3-L1 cells. Western blotting detected increased TAZ protein levels in 3T3-L1 cells that were transfected with the TAZ expression plasmid (Fig. $5 A)$. Treatment with sclerostin failed to detect any significant increases of oil red $O$ staining and adiponectin mRNA expression in TAZ over-expressed cells (Figs. 5B, 5C and 5D). These results indicate that adipocyte differentiation is TAZ dependent and that sclerostin may be involved in regulating adipocyte differentiation via TAZ. 


\section{Discussion}

In this study, osteocyte-produced sclerostin enhances adipocyte differentiation in 3 T3-L1 preadipocytes. Adipose tissue mass is determined by the increase in adipocyte size and number [Ali et al., 2013]. The size of adipocytes augments because of increased storage of triacylglycerols from dietary sources or endogenous lipogenesis. On the other hand, adipocyte number increases as a result of enhanced cell proliferation and differentiation [Ali et al., 2013]. Evidence from several in vivo studies supports the idea that sclerostin regulates adipose tissue. For example, it has been shown that serum circulating sclerostin is related to fat metabolism and adiposity [Amrein et al., 2012; Colaianni et al., 2014; Urano et al., 2012]. Recently, Ma et al. reported a cross-sectional cohort study showing that serum sclerostin was positively associated with total fat mass [Ma et al., 2014]. Adipocyte differentiation therefore requires the cells to process a variety of combinatorial inputs during differentiation induction. Identification of various molecules that modulate the process in either a positive or negative manner provides insight into the fat metabolism regulation in the adipose tissue [Ali et al., 2013]. With our study we provide a molecular novel role for osteocyte-produced sclerostin in metabolism control between adipose tissue and bone tissue. We speculate that osteocyte lacunocanalicular network can function as an endocrine system to secrete sclerostin into blood targeting distant organs. To date, there are few reports on the regulation of sclerostin expression in osteocytes and on its entry into the circulation. Since adipose tissue produces a variety of secretory factors that exert effects at the systemic level, such factors may regulate sclerostin production and secretion from the bone tissue.

During adipocyte differentiation, sclerostin may act via specific receptors to transduce external growth and differentiation signals through a cascade of intracellular events. Sclerostin binds to LRP5/6, and point mutations in the amino-terminal 
$\beta$-propeller domain of LRP5, which are associated with high bone mass, reduce the ability of sclerostin to interact with LRP5 [Semënov et al., 2005; Semenov and He, 2006], suggesting that sclerostin interacts with the amino-terminal region of LRP $5 / 6$, thus mediating biological functions. Sclerostin has also been shown to bind to another member of the LDL receptor family, LRP4 [Choi et al., 2009], and different regions of sclerostin interact with LRP5/6 and LRP4 [Holdsworth et al., 2012]. LRP4/5/6 are widely and constitutively expressed in several types of peripheral tissues including osteoblasts [He et al., 2004]. A rare mutation in LRP6 was found to be associated with a metabolic syndrome and with diabetes [Mani et al., 2007; Singh et al., 2013]. $L R P 6^{+/-}$mice on a high fat diet were protected against diet-induced obesity and adipose tissue insulin resistance compared with their wild-type littermates, suggesting that LRP6 regulates genes involved in adipogenesis, metabolism and insulin signaling [Liu et al., 2012]. Our study shows that 3T3-L1 preadopocytes express detectable levels of LRP5/6 but not of LRP4, suggesting that sclerostin acts via LRP5/6 to transduce signals through a cascade of intracellular events during adipocyte differentiation. Since LRP6 has been shown to regulate body weight and glucose metabolism as a nutrient sensing factor, we think that sclerostin may have a role in nutrient sensing. Although, since LRP dominant function during adipocyte differentiation regulated by sclerostin is unknown, our observation supports the idea that LRP5/6 is predominantly expressed by preadipogenic cells and mediates adipocyte differentiation interacting with sclerostin in the adipose tissue.

Sclerostin is known as an inhibitor of the canonical Wnt signaling pathway. Signaling ligands such as Wnt1 or Wnt10b suppress adipocyte differentiation [Bennett et al., 2002; Ross et al., 2000]. Based on our observation, not only sclerostin but also the small molecular inhibitors IWR-1 may block the activation of canonical Wnt signaling pathway [Chen et al., 2009] induced by adipocyte differentiation in 3T3-L1 cells (data 
not shown). Consistent with our studies, it has been shown that dominant-negative TCF4 or another soluble inhibitor of Wnt signaling, such as the secreted frizzled related protein, induces adipocyte differentiation [Bennett et al., 2002; Ross et al., 2000]. Taken together, our results suggest that sclerostin may inhibit endogenous canonical Wnt signaling and then enhance adipocyte differentiation. Another possibility is that sclerostin itself may interact with specific receptors and induce certain intracellular signaling, resulting in 3T3-L1 adipocyte differentiation. With this work we have uncovered a precise molecular mechanism by which sclerostin may function as an inducer of adipocyte differentiation.

During 3T3-L1 adipocyte differentiation, C/EBP $\beta$ is induced early and plays a crucial role [Guo et al., 2015]. Upon the treatment with differentiation inducer, growth-arrested 3T3-L1 cells re-enter the cell cycle, a process referred to as mitotic clonal expansion (MCE) characterized by impaired proliferation, which contributes to adipocyte hyperplasia. The adipogenic gene expression program is initiated during and after MCE, ultimately leading to terminal adipocyte differentiation [Guo et al., 2015]. Several lines of evidence have shown that C/EBP $\beta$ is involved during MCE. Enhanced expression of C/EBP $\beta$ by sclerostin-as shown by our results-may contribute to MCE, resulting in the enhancement of 3T3-L1 adipocyte differentiation.

TAZ, a transcriptional modulator, has a key role in cell proliferation, differentiation, and stem cell self-renewal. TAZ activity is regulated by several signaling pathways, including Hippo and canonical Wnt signaling [Piccolo et al., 2014]. In this study, we show that sclerostin inactivates the TAZ responsive luciferase reporter containing responsive elements TEAD, which is induced by Wnt3a. Recently, Byun et al. [Byun et al., 2014] reported that Wnt3a facilitates the dephosphorylation of TAZ, stabilizing TAZ and preventing its binding to 14-3-3 proteins, thus inducing nuclear localization of TAZ. Our analysis implies the presence of a transcriptional machinery 
that is sensitive to sclerostin, that regulates TAZ activity, and that modulates transcriptional activity through interaction with the TAZ-responsive gene promoter (i.e., ctgf gene) [Zhang et al., 2009]. Recently, in human clinical studies, the administration of sclerostin-neutralizing monoclonal antibodies has shown that pharmacologic inhibition of sclerostin results in increased bone formation, bone mass, and bone strength [McClung et al., 2014]. Sclerostin upregulates adipocyte differentiation, suggesting that an anti-sclerostin neutralizing antibody might act as a potent TAZ activator and could be an anabolic agent to be used therapeutically to prevent or reverse fat gain in conditions such as metabolic diseases.

In conclusion, we have shown that sclerostin regulates adipocyte differentiation. This is the first molecular study linking the osteocyte-derived molecule sclerostin to adipocytes. Further investigations may provide important new information pertaining to the molecular basis of the cross-regulation of metabolism between bone and fat tissues. 


\section{References}

Azzolin L, Panciera T, Soligo S, Enzo E, Bicciato S, Dupont S, Bresolin S, Frasson C, Basso G, Guzzardo V, Fassina A, Cordenonsi M, Piccolo S. 2014. YAP/TAZ incorporation in the $\beta$-catenin destruction complex orchestrates the Wnt response. Cell 158:157-70.

Ali AT, Hochfeld WE, Myburgh R, Pepper MS. 2013. Adipocyte and adipogenesis. Eur J Cell Biol 92:229-36.

Amrein K, Amrein S, Drexler C, Dimai HP, Dobnig H, Pfeifer K, Tomaschitz A, Pieber TR, Fahrleitner-Pammer A. 2012. Sclerostin and its association with physical activity, age, gender, body composition, and bone mineral content in healthy adults. J Clin Endocrinol Metab 97:148-54.

Azzolin L, Zanconato F, Bresolin S, Forcato M, Basso G, Bicciato S, Cordenonsi M, Piccolo S. 2012. Role of TAZ as mediator of Wnt signaling. Cell 151:1443-56.

Balemans W, Ebeling M, Patel N, Van Hul E, Olson P, Dioszegi M, Lacza C, Wuyts W, Van Den Ende J, Willems P, Paes-Alves A, Hill S, Bueno M, Ramos F, Tacconi P, Dikkers F, Stratakis C, Lindpaintner K, Vickery B, Foernzler D, Van Hul W. 2001. Increased bone density in sclerosteosis is due to the deficiency of a novel secreted protein (SOST). Hum Mol Genet 10:537-43.

Baron R, Kneissel M. 2013. WNT signaling in bone homeostasis and disease: from human mutations to treatments. Nat Med 19:179-92.

Bennett CN, Ross SE, Longo KA, Bajnok L, Hemati N, Johnson KW, Harrison SD, MacDougald OA. 2002. Regulation of Wnt signaling during adipogenesis. J Biol Chem 277:30998-1004.

Burgers TA, Williams BO. 2013. Regulation of Wnt/ß-catenin signaling within and from osteocytes. Bone 54:244-9.

Byun MR, Hwang JH, Kim AR, Kim KM, Hwang ES, Yaffe MB, Hong JH. 2014. Canonical Wnt signalling activates TAZ through PP1A during osteogenic differentiation. Cell Death Differ 21:854-63.

Chen B, Dodge ME, Tang W, Lu J, Ma Z, Fan CW, Wei S, Hao W, Kilgore J, Williams NS, Roth MG, Amatruda JF, Chen C, Lum L. 2009. Small molecule-mediated disruption of Wnt-dependent signaling in tissue regeneration and cancer. Nat Chem Biol 5:100-7. 
Choi HY, Dieckmann M, Herz J, Niemeier A. 2009. Lrp4, a novel receptor for Dickkopf 1 and sclerostin, is expressed by osteoblasts and regulates bone growth and turnover in vivo. PLoS One 4:e7930.

Colaianni G, Brunetti G, Faienza MF, Colucci S, Grano M. 2014. Osteoporosis and obesity: Role of Wnt pathway in human and murine models. World J Orthop 5:242-6.

DiGirolamo DJ, Clemens TL, Kousteni S. 2012. The skeleton as an endocrine organ. Nat Rev Rheumatol 8:674-83.

Dupont S, Morsut L, Aragona M, Enzo E, Giulitti S, Cordenonsi M, Zanconato F, Le Digabel J, Forcato M, Bicciato S, Elvassore N, Piccolo S. 2011. Role of YAP/TAZ in mechanotransduction. Nature 474:179-83.

Ferron M, Hinoi E, Karsenty G, Ducy P. 2008. Osteocalcin differentially regulates beta cell and adipocyte gene expression and affects the development of metabolic diseases in wild-type mice. Proc Natl Acad Sci U S A 105:5266-70.

Guo L, Li X, Tang QQ. 2015. Transcriptional regulation of adipocyte differentiation: a central role for CCAAT/enhancer-binding protein (C/EBP) $\beta$. J Biol Chem 290:755-61.

He X, Semenov M, Tamai K, Zeng X. 2004. LDL receptor-related proteins 5 and 6 in Wnt/beta-catenin signaling: arrows point the way. Development 131:1663-77.

Holdsworth G, Slocombe P, Doyle C, Sweeney B, Veverka V, Le Riche K, Franklin RJ, Compson J, Brookings D, Turner J, Kennedy J, Garlish R, Shi J, Newnham L, McMillan D, Muzylak M, Carr MD, Henry AJ, Ceska T, Robinson MK. 2012. Characterization of the interaction of sclerostin with the low density lipoprotein receptor-related protein (LRP) family of Wnt co-receptors. J Biol Chem 287:26464-77.

lizuka S, Oridate N, Nashimoto M, Fukuda S, Tamura M. 2014. Growth Inhibition of Head and Neck Squamous Cell Carcinoma Cells by sgRNA Targeting the Cyclin D1 mRNA Based on TRUE Gene Silencing. Plos One 9.

Kadowaki T, Yamauchi T. 2005. Adiponectin and adiponectin receptors. Endocr Rev 26:439-51.

Kajimura D, Lee HW, Riley KJ, Arteaga-Solis E, Ferron M, Zhou B, Clarke CJ, Hannun YA, DePinho RA, Guo XE, Guo EX, Mann JJ, Karsenty G. 2013. Adiponectin regulates bone mass via opposite central and peripheral mechanisms through FoxO1. Cell Metab 17:901-15. 
Kanai F, Marignani PA, Sarbassova D, Yagi R, Hall RA, Donowitz M, Hisaminato A, Fujiwara T, Ito Y, Cantley LC, Yaffe MB. 2000. TAZ: a novel transcriptional co-activator regulated by interactions with 14-3-3 and PDZ domain proteins. EMBO J 19:6778-91.

Ke HZ, Richards WG, Li X, Ominsky MS. 2012. Sclerostin and Dickkopf-1 as therapeutic targets in bone diseases. Endocr Rev 33:747-83.

Karsenty G, Oury F. 2012. Biology without walls: the novel endocrinology of bone. Annu Rev Physiol 74:87-105.

Klangjareonchai T, Nimitphong $H$, Saetung $S$, Bhirommuang $N$, Samittarucksa $R$, Chanprasertyothin S, Sudatip R, Ongphiphadhanakul B. 2014. Circulating sclerostin and irisin are related and interact with gender to influence adiposity in adults with prediabetes. Int J Endocrinol 2014:261545.

Kramer I, Loots GG, Studer A, Keller H, Kneissel M. 2010. Parathyroid hormone (PTH)-induced bone gain is blunted in SOST overexpressing and deficient mice. $J$ Bone Miner Res 25:178-89.

Liu W, Singh R, Choi CS, Lee HY, Keramati AR, Samuel VT, Lifton RP, Shulman GI, Mani A. 2012. Low density lipoprotein (LDL) receptor-related protein 6 (LRP6) regulates body fat and glucose homeostasis by modulating nutrient sensing pathways and mitochondrial energy expenditure. J Biol Chem 287:7213-23.

Li X, Ominsky MS, Niu QT, Sun N, Daugherty B, D'Agostin D, Kurahara C, Gao Y, Cao J, Gong J, Asuncion F, Barrero M, Warmington K, Dwyer D, Stolina M, Morony S, Sarosi I, Kostenuik PJ, Lacey DL, Simonet WS, Ke HZ, Paszty C. 2008. Targeted deletion of the sclerostin gene in mice results in increased bone formation and bone strength. J Bone Miner Res 23:860-9.

Li X, Zhang Y, Kang H, Liu W, Liu P, Zhang J, Harris SE, Wu D. 2005. Sclerostin binds to LRP5/6 and antagonizes canonical Wnt signaling. J Biol Chem 280:19883-7.

MacDougald OA, Lane MD. 1995. Transcriptional regulation of gene expression during adipocyte differentiation. Annu Rev Biochem 64:345-73.

Mani A, Radhakrishnan J, Wang H, Mani MA, Nelson-Williams C, Carew KS, Mane S, Najmabadi $H$, Wu D, Lifton RP. 2007. LRP6 mutation in a family with early coronary disease and metabolic risk factors. Science 315:1278-82.

Ma YH, Schwartz AV, Sigurdsson S, Hue TF, Lang TF, Harris TB, Rosen CJ, Vittinghoff E, Eiriksdottir G, Hauksdottir AM, Siggeirsdottir K, Sigurdsson G, Oskarsdottir D, Napoli N, Palermo L, Gudnason V, Li X. 2014. Circulating sclerostin associated with 
vertebral bone marrow fat in older men but not women. $J$ Clin Endocrinol Metab 99:E2584-90.

McClung MR, Grauer A, Boonen S, Bolognese MA, Brown JP, Diez-Perez A, Langdahl BL, Reginster JY, Zanchetta JR, Wasserman SM, Katz L, Maddox J, Yang YC, Libanati C, Bone HG. 2014. Romosozumab in postmenopausal women with low bone mineral density. N Engl J Med 370:412-20.

MacDonald B, Tamai K, He X. 2009. Wnt/beta-catenin signaling: components, mechanisms, and diseases. Dev Cell 17:9-26.

Nakashima A, Katagiri T, Tamura M. 2005. Cross-talk between Wnt and bone morphogenetic protein 2 (BMP-2) signaling in differentiation pathway of C2C12 myoblasts. J Biol Chem 280:37660-37668.

Piccolo S, Dupont S, Cordenonsi M. 2014. The biology of YAP/TAZ: hippo signaling and beyond. Physiol Rev 94:1287-312.

Rosen ED, Spiegelman BM. 2000. Molecular regulation of adipogenesis. Annu Rev Cell Dev Biol 16:145-71.

Ross SE, Hemati N, Longo KA, Bennett CN, Lucas PC, Erickson RL, MacDougald OA. 2000. Inhibition of adipogenesis by Wnt signaling. Science 289:950-3.

Semënov M, Tamai K, He X. 2005. SOST is a ligand for LRP5/LRP6 and a Wnt signaling inhibitor. J Biol Chem 280:26770-5.

Semenov MV, He X. 2006. LRP5 mutations linked to high bone mass diseases cause reduced LRP5 binding and inhibition by SOST. J Biol Chem 281:38276-84.

Singh R, De Aguiar RB, Naik S, Mani S, Ostadsharif K, Wencker D, Sotoudeh M, Malekzadeh R, Sherwin RS, Mani A. 2013. LRP6 enhances glucose metabolism by promoting TCF7L2-dependent insulin receptor expression and IGF receptor stabilization in humans. Cell Metab 17:197-209.

Takeda S, Elefteriou F, Levasseur R, Liu X, Zhao L, Parker KL, Armstrong D, Ducy P, Karsenty G. 2002. Leptin regulates bone formation via the sympathetic nervous system. Cell 111:305-17.

Urano T, Shiraki M, Ouchi Y, Inoue S. 2012. Association of circulating sclerostin levels with fat mass and metabolic disease--related markers in Japanese postmenopausal women. J Clin Endocrinol Metab 97:E1473-7.

Uyama M, Sato MM, Kawanami M, Tamura M. 2012. Regulation of osteoblastic differentiation by the proteasome inhibitor bortezomib. Genes To Cells 17:548-558. 
van Bezooijen R, ten Dijke P, Papapoulos S, Löwik C. 2005. SOST/sclerostin, an osteocyte-derived negative regulator of bone formation. Cytokine Growth Factor Rev 16:319-27.

Zhang H, Liu CY, Zha ZY, Zhao B, Yao J, Zhao S, Xiong Y, Lei QY, Guan KL. 2009. TEAD transcription factors mediate the function of TAZ in cell growth and epithelial-mesenchymal transition. J Biol Chem 284:13355-62.

Zhang H, Liu CY, Zha ZY, Zhao B, Yao J, Zhao S, Xiong Y, Lei QY, Guan KL. 2009. TEAD transcription factors mediate the function of TAZ in cell growth and epithelial-mesenchymal transition. J Biol Chem 284:13355-62. 


\section{FIGURE LEGENDS}

\section{Fig. 1}

\section{Sclerostin enhances adipocyte differentiation in 3T3-L1 cells}

Confluent 3T3-L1 cells were incubated in differentiation media and treated with indicated doses of sclerostin at day 0 postinitiation of differentiation. Cells were fixed and lipid accumulation was monitored by oil red $O$ staining at day 5 of differentiation $(A)$. Lipid staining was extracted using isopropyl alcohol and oil red $\mathrm{O}$ accumulation quantified by measuring absorbance at $520 \mathrm{~nm}$. Fold-increase in absorbance over nontreated cells is presented (B). After total RNA was extracted from the cells at day 5 of differentiation, adiponectin or peroxisome proliferator-activated receptor (PPAR) $Y$ mRNA level was determined by qRT-PCR $(C)$. The lipoprotein lipase (LPL) and the fatty acid-binding protein 4 (Fabp4) mRNA levels were determined by qRT-PCR (D). $\beta$-actin was used as an endogenous control. Data are presented as means \pm S.D.; $n=3,{ }^{*}, P<$ 0.05 versus absence of sclerostin (0).

\section{Fig. 2}

\section{Sclerostin attenuates Wnt3a-inhibited adipocyte differentiation and expression of}

\section{LRP5 and LRP6 in 3T3-L1 cells}

( $A$ and $B$ ) Confluent 3T3-L1 cells were incubated in differentiation media $(D M+)$ or none (DM -) and treated with indicated doses of sclerostin $(20 \mathrm{ng} / \mathrm{mL})$, Wnt3a $(10 \mathrm{ng} / \mathrm{mL})$, sclerostin $(50 \mathrm{ng} / \mathrm{mL})$ and Wnt3a $(10 \mathrm{ng} / \mathrm{mL})$ or vehicle (-). At day 5 , total RNA was extracted from the cells and adiponectin and PPARY mRNA levels were determined by qRT-PCR. GAPDH was used as an endogenous control. Data are presented as means \pm S.D.; $n=3,{ }^{*}, P<0.05$. (B) Photograph of oil red $O$ staining (left panel) and quantification of oil red $\mathrm{O}$ staining at day 5 (right panel). (C) LRP4, LRP5, and LRP6 mRNA expression were determined by RT-PCR in 3T3-L1 preadipocytes (A) or 
MC3T3-E1 osteoblasts (O). $\beta$-actin was used as a positive control. Lane $\mathrm{M}$ represents the size marker (100-bp ladder).

Fig. 3

Sclerostin regulates C/EBPB expression but not cell proliferation and or caspase-3/7 activity in 3T3-L1 cells

(A) Confluent 3T3-L1 cells were incubated in differentiation media and treated with sclerostin $(20 \mathrm{ng} / \mathrm{mL})$. After 24 hours, the levels of C/EBP $\beta$ protein in the cells were determined by western blot analysis. (B) Confluent 3T3-L1 cells were incubated in differentiation media and treated with sclerostin $(20 \mathrm{ng} / \mathrm{mL})$, and then cultured for further $24 \mathrm{~h}$. DNA synthesis of 3T3-L1 cells was measured by BrdU incorporation using an ELISA kit. BrdU incorporation in the absence of sclerostin is adjusted to 1. (C) Confluent 3T3-L1 cells were incubated in differentiation media and treated with sclerostin (20 $\mathrm{ng} / \mathrm{mL}$ ). After $4 \mathrm{~h}$, cellular caspase-3/7 activities were measured. Fold-increase in activity was calculated based on activity measured in control (absence) cells. Each assay represents a separate experiment performed in triplicate. Data are presented as means $\pm S . D ; n=3 ;$ n.s.(no significant difference)

Fig. 4

Effects of TAZ knockdown on sclerostin-mediated adipocyte differentiation in 3T3-L1 cells

(A) 3T3-L1 cells were incubated in differentiation media and then transiently cotransfected in 24-well plates with a TAZ reporter plasmid 8xGTIIC-Lux. Then cells were treated with sclerostin $(20 \mathrm{ng} / \mathrm{mL})$, Wnt3a $(10 \mathrm{ng} / \mathrm{mL})$, sclerostin $(20 \mathrm{ng} / \mathrm{mL})$ and Wnt3a (10 ng/mL) or vehicle (-) for $6 \mathrm{~h}$, after which luciferase activity was determined. Normalized luciferase activity is shown as the ratio of luciferase activity relative to 
8xGTIIC-Lux with vehicle, which is set to a value of 1. (B) Confluent 3T3-L1 cells were incubated in differentiation media and treated with sclerostin $(20 \mathrm{ng} / \mathrm{mL})$, Wnt3a $(10$ $\mathrm{ng} / \mathrm{mL})$, sclerostin (50 $\mathrm{ng} / \mathrm{mL})$ and Wnt3a $(10 \mathrm{ng} / \mathrm{mL})$ or vehicle (-) for 5 days. Total RNA was extracted from the cells and then ctgf mRNA level was determined by qRT-PCR. (C, D, E, and F) 3T3-L1 cells were transiently transfected with TAZ siRNA (siTAZ) or control siRNA (siCont) (both at $10 \mathrm{nM}$ ) at day 0 . Then cells were treated with sclerostin (20 $\mathrm{ng} / \mathrm{mL}$ ) or vehicle (-). At day 2 , the levels of TAZ protein in the cells were determined by western blot analysis. (D) Photographs of oil red $O$ staining and $(E)$ quantification of oil red $\mathrm{O}$ staining in 3T3-L1 cells at day 6. Graph showing fold-increase in absorbance over nontreated cells. (F) A qRT-PCR was performed to quantify mRNA expression level of adiponectin. $\beta$-actin was used as an endogenous control. Data are presented as means \pm S.D; $\mathrm{n}=3 ;{ }^{*}, \mathrm{P}<0.05$.

Fig. 5

Effects of TAZ over-expression on sclerostin-mediated adipocyte differentiation in 3T3-L1 cells

3T3-L1 cells were transiently transfected in 24-well plates with a TAZ expression plasmid pTAZ or empty vector pcDNA3 (both at $0.1 \mu \mathrm{g} /$ well). After one day, cells were treated with sclerostin $(20 \mathrm{ng} / \mathrm{mL})$ or vehicle (-) (day 0$)$. The levels of TAZ protein in the cells were determined by western blot analysis at day $2(A)$. Photographs of oil red $O$ staining $(B)$ and quantification of oil red $O$ staining $(C)$ in 3T3-L1 cells at day 6 . Total RNA was extracted from the cells at day 6 and then a qRT-PCR was performed to quantify mRNA expression level of adiponectin (D). $\beta$-actin was used as an endogenous control. Data are presented as means $\pm S . D ; n=3$; n.s. (no significant difference) 
Figure 1

A

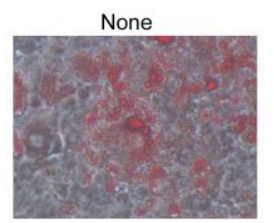

$10 \mathrm{ng} / \mathrm{mL}$

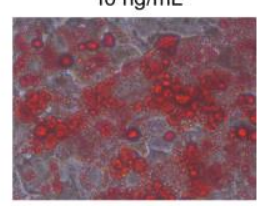

C

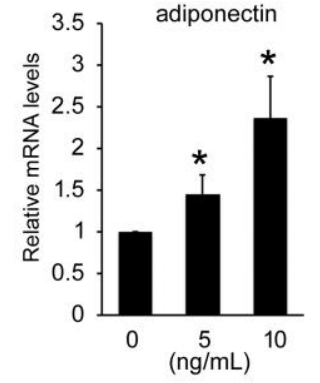

$5 \mathrm{ng} / \mathrm{mL}$

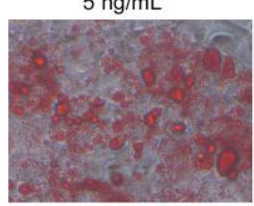

$20 \mathrm{ng} / \mathrm{mL}$
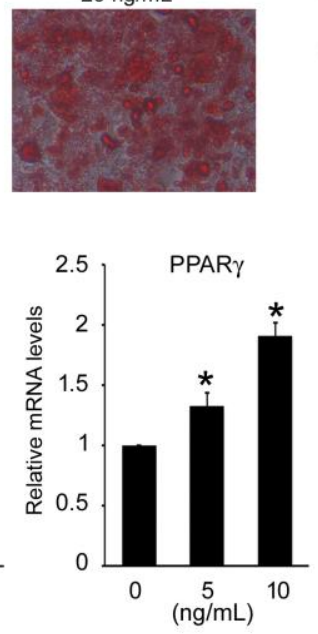

B

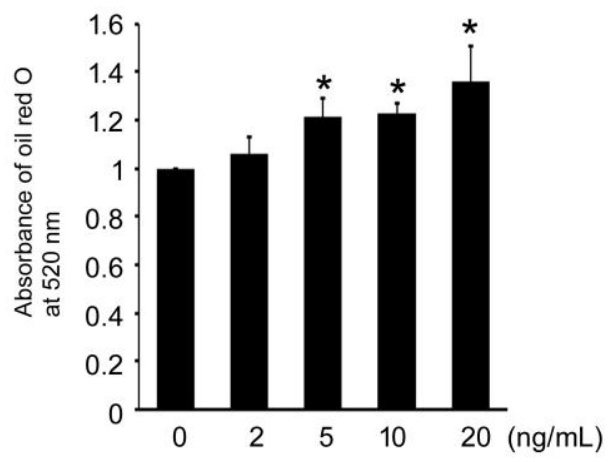

$\begin{array}{llllll}D & 2 & \text { LPL } & 2 & \text { Fabp4 }\end{array}$
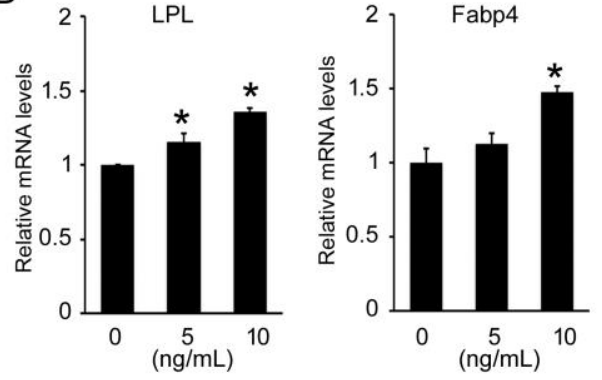
Figure 2

A
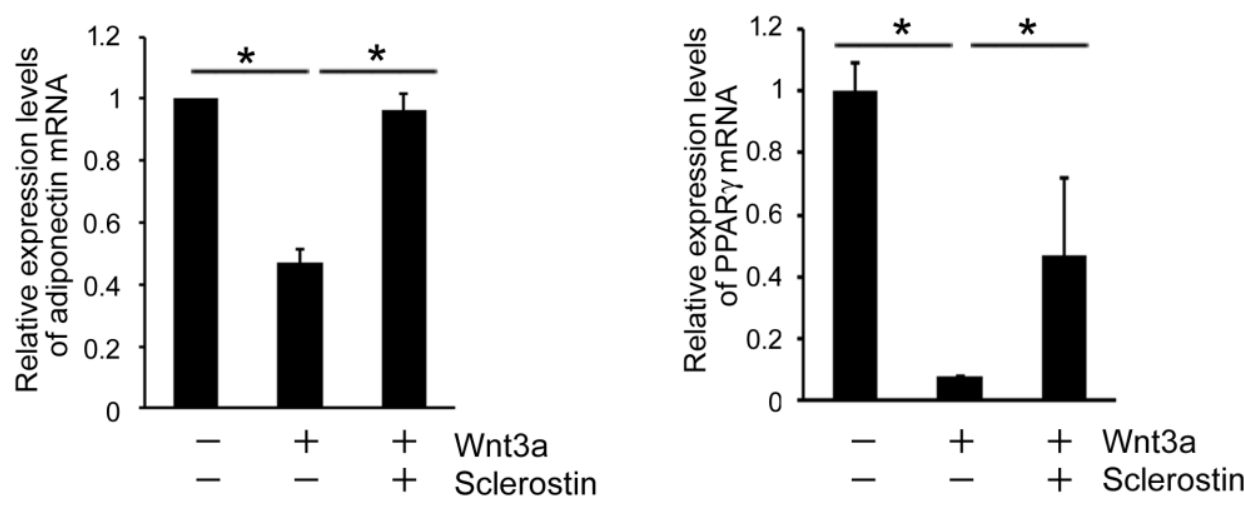

B

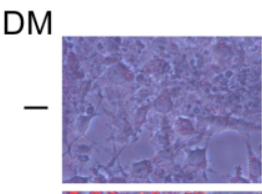

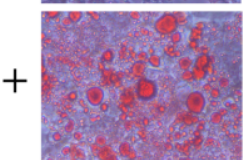

$-$

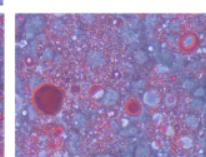

$+$
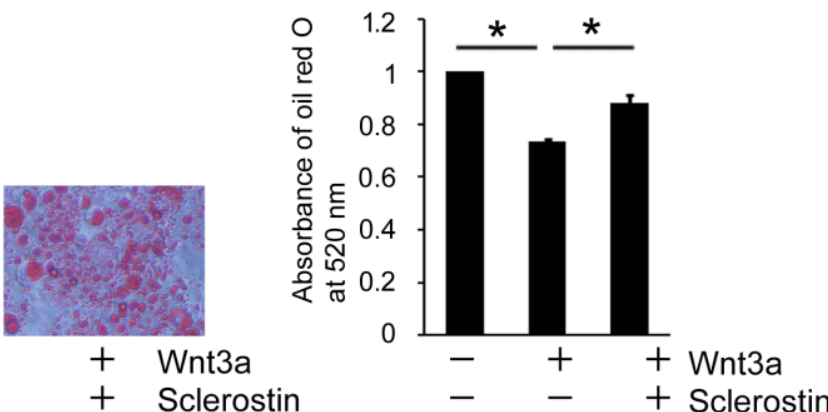

C

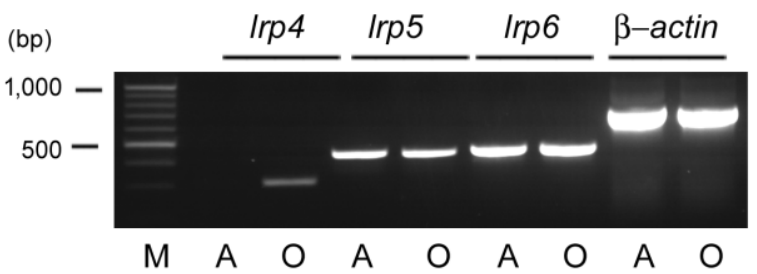


Figure 3

A

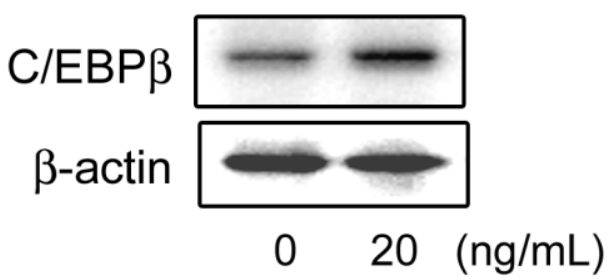

B

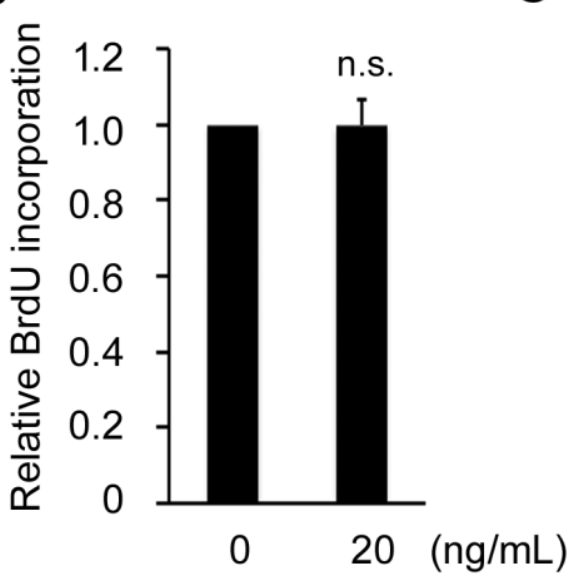

C

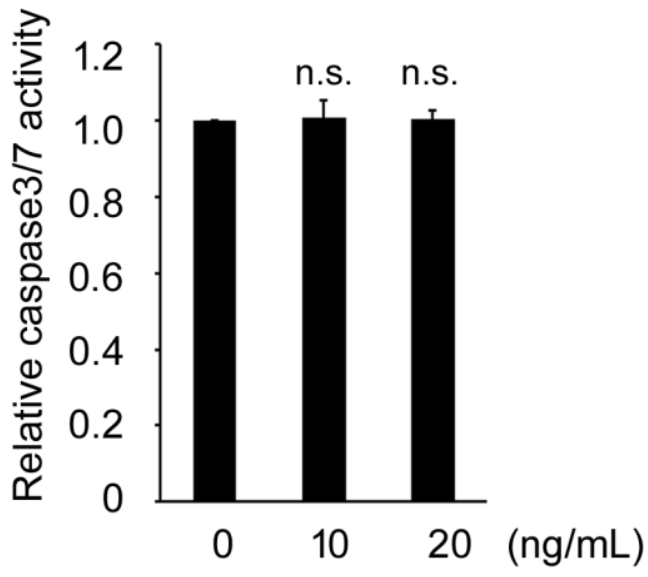


Figure 4

A

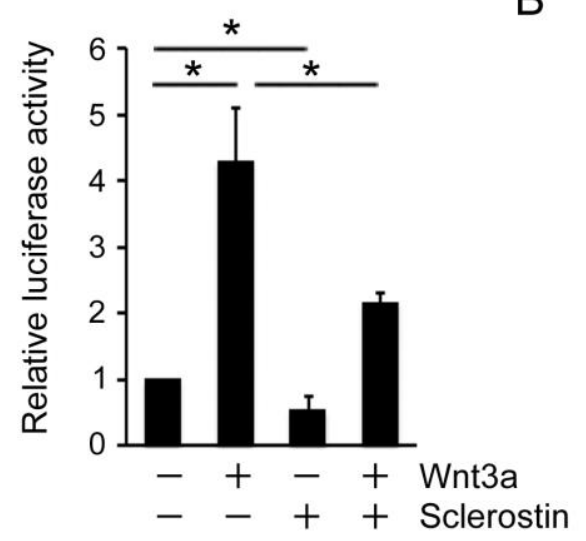

B

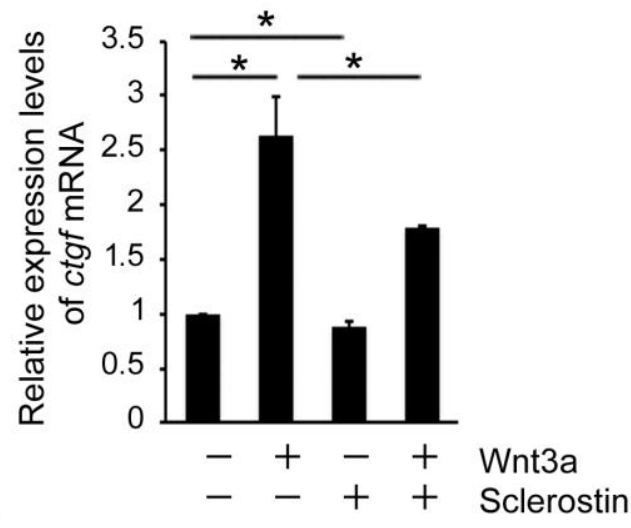

C

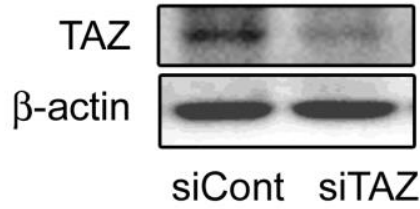

D

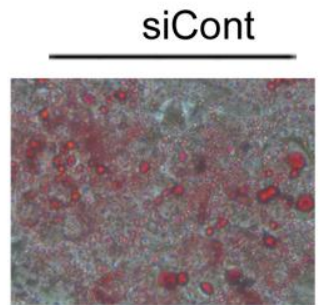

-

E

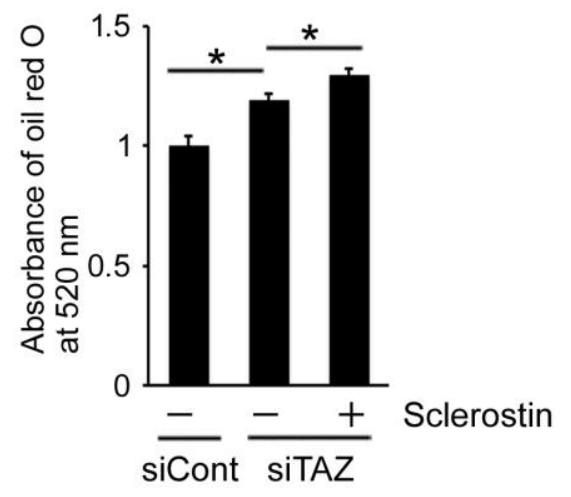

F

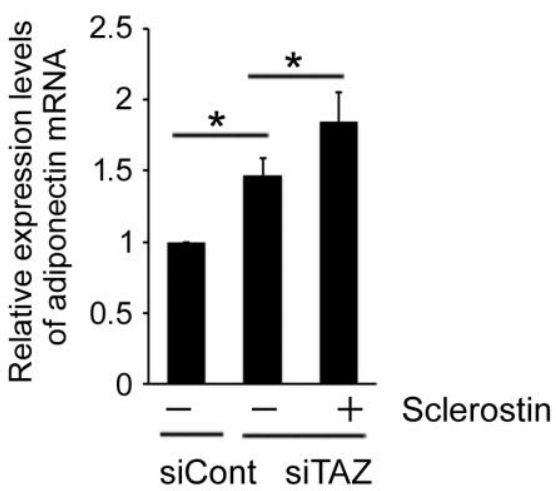


Table 1

Primers used in RT-PCR analysis

\begin{tabular}{llc}
\hline & \multicolumn{1}{c}{ sequence (5' to 3') } & predicted size (bp) \\
\hline LRP4 & AGGACTGCACGTCAGCTATG & 321 \\
& TTGAGGTCACCCCATTCAGC & \\
LRP5 & CCATTGTGTTGCACCCTGTG & 450 \\
& TGCACCCTCCATTTCCATCC & \\
LRP6 & GCAACGATTGTAGTTGGAGGC & 468 \\
& CCAGTAAAGCTTCCGCTCCT & \\
\multirow{2}{*}{-actin } & GTGGGCCGCTCTAGGCACCAA & 540 \\
& GTCTTTGATGTCACGCACGATTTC & \\
\hline
\end{tabular}

